DOI 10.18699/SBB-2020-41

\title{
In vitro evaluation of new nuclease class II type V system
}

\author{
Zheltova A.*, Medvedev D., Polushkina I., Vasiliev R. \\ National Research Centre "Kurchatov Institute", Moscow, Russia \\ *e-mail: annettezv@gmail.com
}

Key words: CRISPR-Cas, nucleases, Cas12, Cpf1

Motivation and Aim: Adaptive immunity systems of prokaryotes CRISPR-Cas consist of Clustered Regularly Interspaced Short Palindromic Repeats (CRISPR) and CRISPR-associated proteins (Cas). CRISPR systems have been grouped into six different types (I-VI), which are divided into two large classes (I and II) [1]. One of the most widely used V type proteins is the Cpf1 (Cas12a). It has been shown that genome editing with CRISPR-Cas is a useful tool for genetic engineering. For example, Cpf1 orthologs such as Acidaminococcus sp. Cpfl (AsCpf1) show stable activity in mammalian cells and have been used to edit the genome of eukaryotic mammalian cells [2]. Nevertheless, there is an ongoing need for the search and development of new nucleases with improved definition of sequence-specific nucleic acid, its cleavage, and the ability to manipulate in various experimental conditions. The objective of this study was to carry out an in vitro evaluation of a new nuclease class II type $\mathrm{V}$.

Methods and Algorithms: Bioinformatic search made it possible to find and evaluate protein for in vitro testing. The nuclease sequence was obtained from the metagenome samples using PCR, and then cloning into a plasmid was carried out. The recombinant protein was obtained and purified using a Ni-NTA metal chelate column and cation exchange chromatography. To determine the activity of nuclease, experiments of in vitro cleavage under various conditions were performed; to assess the success of cleavage, electrophoresis was used.

Results: By bioinformatic search in metagenomic data, new effector Cas proteins were discovered. Bioinformatic evaluation was performed and the optimal protein for in vitro validation was selected. It was shown that this nuclease is an effector enzyme of the class II subtype V-A CRISPR-Cas. In addition, this nuclease is smaller than AsCpf. To determine the functional activity of new nuclease, various cleavage buffers were analyzed and the optimal one was selected (the experiment was carried out at 37 degrees for 30 minutes). Next, the time required for DNA cleavage was determined: there was no difference between 5 and 60 minutes. It was found some difference between efficiency of cleavage using full and split gRNA $(20+20)$. Also, with an increase in the concentration of protein and RNA, a dose-dependent cleavage of the DNA substrate was established. It was tested whether the cleavage of DNA by nuclease is temperature dependent. Incubation of nuclease, gRNA, and DNA template, carrying a protospacer flanked by the PAM sequence, at various temperatures resulted in efficient cleavage of the target in the temperature range 8-80 ${ }^{\circ} \mathrm{C}$ with maximum cleavage at 40 and $50{ }^{\circ} \mathrm{C}$.

Conclusion: A new active RNA-guided nuclease that efficiently introduces double-stranded breaks in target DNA was discovered and characterized.

\section{References}

1. Makarova K.S., Wolf Y.I., Iranzo J. et al. Evolutionary classification of CRISPR-Cas systems: a burst of class 2 and derived variants. Nat Rev Microbiol. 18, 67-83 (2020).

2. Zetsche B., Gootenberg J.S., Abudayyeh O.O. et al. Cpf1 is a single RNA-guided endonuclease of a class 2 CRISPRCas system. Cell. 2015;163(3):759-771. 